\title{
Promotion of fruits and vegetables in countries of sub-Saharan Africa and the Indian Ocean
}

In a very recent editorial, we gave a report on the increasing importance of chronic diseases such as obesity, diabetes, cardiovascular diseases and cancers, and on the now recognized interest in reinforcing the place of fruits and vegetables in the diet to face these true "epidemics".

These diseases are not a monopoly of industrial countries. They also now concern the countries of the South, especially in urban environments where the nutritional transition is critical. According to the World Health Organization (WHO), the number of diabetics will increase by $170 \%$ in these countries by 2025 . Many factors contribute to a fast increase in these new forms of malnutrition. Greater awareness and multilevel actions are required for addressing these trends. They must be integrated into agriculture, food, health and education policies. It is with this objective that 76 experts from 16 African countries and many representatives of international agencies met recently within a workshop organized in Yaoundé, Cameroon, from October 23rd to 25th, 2007, on the initiative of the FAO and WHO, cO-organized by the IRAD and CIRAD and sponsored by GlobalHort and the CTA. At the center of the debates: the promotion of fruits and vegetables in the French-speaking countries of sub-Saharan Africa and the Indian Ocean. Whereas minimal consumption should be $400 \mathrm{~g}$ of fruit and vegetables per day, the inhabitants of the Sahelian countries consume on average ten times less; among the principal restraints are: the pri- ces of these products, their accessibility and their availability, but also their quality, as many fields which require organization and innovation.

It appears essential to merge sectors of intervention. The fields of health, horticulture, transport, environment and education must operate together. FAO, WHO and GlobalHort initiatives are jointly acting in that direction.

The goal is to mobilize around concrete actions the political representatives of these countries, but also the producers, the fruit and vegetable stakeholders, research, health and education professionals and, of course, funding agencies. In order to maintain the dynamics engaged, annual workshops will be organized in each place through innovation platforms where all the implied stakeholders will be able to exchange and thus promote these productions with a double stake: economic growth through their added value, and public health through their nutritional value.

Dr. Jacky Ganry Chairman of the Section on Tropical and Subtropical Fruits ISHS 


\section{Éditorial}

\section{La promotion des fruits et légumes dans les pays d'Afrique subsaharienne et de l'Océan Indien}

Dans un tout récent éditorial, nous faisions état de l'importance croissante des maladies chroniques comme obésité, diabète, maladies cardio-vasculaires, cancers, et de l'intérêt maintenant reconnu de renforcer la place des fruits et légumes dans la diète alimentaire pour contrecarrer ces véritables "épidémies ".

Ces maladies ne sont pas le monopole des pays développés. Elles concernent désormais aussi les pays du Sud, surtout dans les zones urbaines fortement exposées au phénomène de transition nutritionnelle. D'après l'Organisation mondiale de la santé (OMS), le nombre de diabétiques augmentera de $170 \%$ dans ces pays d'ici 2025. Nombre de facteurs concourent à une augmentation rapide de ces nouvelles formes de malnutrition. Les enrayer implique une prise de conscience et des actions à plusieurs niveaux, intégrées dans les politiques agricoles et alimentaires, de santé et d'éducation. C'est dans cet objectif que 76 experts issus de 16 pays africains et de nombreux représentants d'organismes internationaux se sont réunis récemment au sein d'un atelier organisé à Yaoundé, Cameroun du 23 au 25 octobre 2007, à l'initiative de la FAO et de l'OMS, coorganisé par l'IRAD et le CIRAD et sponsorisé par GlobalHort et le CTA. Au cour des débats : la promotion des fruits et des légumes dans les pays francophones d'Afrique subsaharienne et de l'océan Indien. Alors que la consommation minimale devrait être de $400 \mathrm{~g}$ de fruits et légumes par jour, les habitants des pays sahéliens en consomment en moyenne dix fois moins. Parmi les freins principaux : le prix des ces produits, leur accessibilité, leur disponibilité, mais aussi leur qualité, autant de domaines qui requièrent organisation et innovation.

Il apparaît indispensable de décloisonner les secteurs d'intervention. Les domaines de la santé, de l'horticulture, des transports, de l'environnement et de l'éducation doivent opérer ensemble. Les initiatives mondiales de la FAO, de l'OMS et de GlobalHort s'allient en ce sens.

L'objectif est de mobiliser les représentants politiques de ces pays, mais aussi les producteurs, les acteurs des filières, la recherche, les professionnels de la santé et de l'éducation et, bien sûr, les bailleurs de fonds, autour d'un plan d'action. Afin de maintenir la dynamique engagée, des ateliers annuels seront organisés dans chaque région au travers de plateformes d'innovation où tous les acteurs impliqués pourront échanger et ainsi promouvoir ces productions autour d'un double enjeu : économique de par leur valeur ajoutée, et de santé publique de par leur valeur nutritionnelle.

Dr. Jacky Ganry

Président de la section Fruits tropicaux et subtropicaux

ISHS 